RUidíAZ GARCíA, C., «Percepciones ciudadanas sobre el Sistema Público de los Servicios Sociales en La Rioja», REDUR

8, diciembre 20I0, págs. I47-I67. ISSN i695-078X

\title{
PERCEPCIONES CIUDADANAS SOBRE EL SISTEMA PÚBLICO DE SERVICIOS SOCIALES EN LA RIOJA*
}

\author{
Carmen Ruidíaz GARCÍA \\ PROFESORA TITULAR DE TRABAJO SOCIAL \\ UNIVERSIDAD DE LA RIOJA
}

SUMARIO: Introducción. II. Sistema público de Servicios Sociales. Peculiaridades de un sector emergente de la Política social en España. II.I. De lo general a lo concreto. Algunas consideraciones sobre el Sistema Público Riojano de Servicios Sociales. III. Actitudes y opiniones sobre el Sistema público de Servicios Sociales en La Rioja. III.r. Valoración de Sistema público de Servicios Sociales. III.2. Conocimiento del marco normativo en el que se asientan los Servicios Sociales en la Comunidad Autónoma de La Rioja. III.3. Experiencias personales, directas o indirectas, relacionadas con los Sociales de primer nivel y segundo nivel. III.4. Valoración de las fuentes de financiación del sistema público de Servicios Sociales. III.5. Responsabilidades públicas, privadas, ONGs, etc. en la gestión de los Servicios Sociales, ¿quién lo hace mejor?. III.6. Análisis comparado del sistema y propuestas de mejora. IV. Conclusiones. V. Bibliografía.

RESUMEN: El Sistema público de Servicios Sociales es un sector de la Política social de configuración relativamente reciente. En nuestro país, y por añadidura en La Rioja, en las últimas décadas los Servicios Sociales han crecido de forma considerable tanto en extensión como en contenido. En este trabajo, básicamente descriptivo, realizaré un breve repaso al desarrollo histórico del Sistema público de Servicios Sociales en España y, particularmente, en La Rioja; analizaré las percepciones - actitudes y opiniones - de la población riojana sobre el Sistema Público de Servicios Sociales en la Comunidad autónoma en base a la explotación de los discursos de grupos de discusión realizados a una muestra significativa de la población riojana. En el apartado de conclusiones intentaré condensar la información y realizaré propuestas de desarrollo futuro.

PALABRAS CLAVE: Sistema de bienestar, Política Social, Servicios Sociales, Opinión pública.

KEYWORDS: well-being system, Social Politics, Social Services, public Opinion

\section{Introducción}

Un aspecto importante en los actuales Estados Sociales y Democráticos de Derecho es tantear, mediante la investigación social cualitativa o cuantitativa, a la opinión

* En este artículo he querido resumir una parte del estudio más amplio titulado "Políticas de Servicios Sociales en La Rioja. Descripción, análisis, evaluación y prospectiva desde una perspectiva comparada”, una investigación desarrollada gracias al apoyo económico de la Universidad de la Rioja I+D - API o8 / o9.

La redacción de estas líneas la he realizado en la Universidad Carlos III de Madrid, Dpto. de Ciencia Política y Sociología, durante mi estancia de investigación en el año 20ıo, invitada por la Profa. Dra. Da Mercedes Pardo Buendía 
pública con objeto conocer la visión - actitudes y opiniones - ${ }^{\mathrm{I}}$ que tienen los ciudadanos sobre las políticas públicas tanto globales como sectoriales.

La producción científica sobre el Estado de Bienestar ha puesto de manifiesto que las instituciones y las políticas influyen en la configuración de las actitudes ciudadanas hacia el sistema de bienestar. Expresado de otra manera, cómo se diseñan las políticas, bajo qué perfil ideológico, cómo se implementan, qué estructuras organizativas soportan, grado de implicación de la ciudadanía en el diseño y control de resultados, etc. es determinante en la creación de estereotipos sociales - corrientes o estados de opinión - sobre la acción del Estado en su función de provisor del bienestar colectivo.

Esta misma literatura ha demostrado que, en el caso de España, en nuestra más reciente historia, son las Comunidades Autónomas quienes organizan el Sistema público de bienestar. Este hecho no puede pasar desapercibido dado que existen importantes diferencias en los modelos de provisión autonómicos; en consecuencia, es factible suponer que este mapa autonómico configure actitudes ciudadanas distintas hacia las políticas de bienestar en los diferentes territorios. ${ }^{2}$

Por mi parte, y sin pretensión de abarcarlo todo, en este artículo voy a centrar mi discurso en el análisis de la opinión pública riojana sobre la política pública de Servicios Sociales: resultados percibidos, limitaciones y tensiones que destacan los ciudadanos, valoración de los instrumentos de intervención utilizados por la administración pública, grado en el que el Sistema público atiende las necesidades y demandas cambiantes de los ciudadanos, entre otros aspectos.

Para realizar este análisis voy a explotar la información suministrada en seis grupos de discusión realizados durante el primer trimestre de 20 io a una muestra significativa de la población riojana, hombres y mujeres mayores de i 8 años ${ }^{3}$. Pero antes de pasar a revisar los discursos sociales, voy a presentar, de forma muy resumida, algunos rasgos característicos del Sistema público de Servicios Sociales en España y en La Rioja.

\footnotetext{
En Redur núm. 4 "Notas de investigación. La opinión pública en la Rioja. Aproximación a nuestra epidermis social” me interesé en perfilar el concepto de opinión pública.

Ver http://www.unirioja.es/dptos/dd/redur/numero4.htm

${ }^{2}$ En la actualidad disponemos de interesantes investigaciones sobre actitudes hacia las Políticas sociales desde una perspectiva comparada. Por ejemplo, Gallego, Gomà, Subirats, Subirats (editores.), 2003; Arriba, Calzada y del Pino, 2006, estudios cuantitativos recientes, tal es el caso del número 2829 del Centro de Investigaciones Sociológicas

http://www.cis.es/cis/opencms/ES/I_encuestas/catalogo.html, o el estudio transcultural Actitudes hacia el Bienestar en una Europa cambiante. 2007-2009 Participante experto: Luis Moreno (UPC-CSIC). Investigador responsable: Stefan Svallfors (Universidad de Umea) del que ya se empiezan a presentar resultados en Congresos y Reuniones científicas.

${ }^{3}$ Composición de la muestra: G.D.r: Logroño, hombres de I8 a 25 años, de status medio-bajo-alto. G.D.2: Logroño, mujeres de I8 a 25 años, de estatus medio-bajo-alto. G.D.3. Logroño, hombres de 26 a 55 años, de status medio-bajo-alto. G.D.4. Logroño, mujeres de 26 a 55 años, de status medio-bajo, alto. G.D.5. Resto de La Rioja, hombres de 18 a 30 años, de status medio-bajo. G.D.6. Resto de La Rioja, mujeres de 18 a 30 años, de status medio-medio-alto.

La técnica del grupo de discusión me ha permitido captar los discursos, representaciones ideológicas y las percepciones presentes en colectivos reducidos que comparten ciertas características sociales, haciendo emerger tanto las visiones hegemónicas como los puntos de controversia.

Desde estas páginas, deseo dar las gracias a los miembros de los grupos, ciudadanos anónimos, por su valiosa aportación al estudio así como a D. Jesús García La Hoz por su contribución en la captación de informantes y a D. Alberto Fernández Ruidíaz por el apoyo recibido en la captación de informantes y la transcripción de los grupos de discusión.
} 


\section{Sistema público de Servicios Sociales. Peculiaridades de un sector emergente de la Política social en España}

Desde la creación de los Estados modernos, y anteriormente, la intervención de la Administración en la sociedad ha sido constante y profunda.

En España, la consecuencia directa de la Constitución de I978 ha sido la implantación efectiva del Estado Social y Democrático de Derecho. Este acontecimiento ha supuesto un incremento extraordinario de la intervención de las Administraciones públicas en la sociedad española, tanto en intensidad como en extensión.

Diría más, el Estado social ha supuesto integrar en la sociedad española las concepciones socialdemócratas imperantes en Europa de la postguerra, que no consideraban suficiente crear Estados democráticos de Derecho sino Estados sociales que garantizaran, promovieran, tutelaran, promocionaran, fomentaran, organizaran, etc. el ejercicio de derechos o actividades sociales y económicas de gran relevancia para los ciudadanos. En este marco, la intervención de la Administración en el ámbito de la acción social encuentra su punto de inflexión en el Sistema Público de Servicios Sociales. ${ }^{4}$

Ahora bien, y pese a estas consideraciones de partida, existe un consenso general en las democracias occidentales al considerar a los Servicios Sociales como la extensión de los derechos sociales; unos servicios que derivan de procesos complejos, de dinámicas de carácter discontinuo y de numerosas peculiaridades. En su dimensión objetiva de servicio a los ciudadanos y grupos en que se integran, han existido desde siempre, si bien su nominación como tales sea bastante reciente, posiblemente por la necesidad de diferenciarlos de otros instrumentos que conforman el bienestar social, tales como la Seguridad Social, la Sanidad, la Educación, el Empleo o la Vivienda.

En el caso de España, el Sistema Público de Servicios Sociales debe analizarse como un sistema sometido al Derecho público. Este carácter público se encuentra explícitamente presente en todas las Leyes autonómicas de Servicios Sociales ${ }^{5}$, tanto de

${ }^{4}$ DISERTACIÓN. La existencia de riesgos es un elemento objetivo, por ello, la sociedad debe arbitrar mecanismos de respuesta a ellos a causa de la dimensión social de los problemas.

La conciencia del carácter social de los riesgos encuentra su punto de inflexión con la aparición del capitalismo y con la Revolución Industrial; fue entonces cuando empezó a considerarse que derivaban del propio sistema y se elaboraron técnicas de cobertura específicas, de prevención y reparo, y también técnicas inespecíficas de ahorro, beneficencia, mutualidad y seguro privado. Entre las técnicas específicas, el mutualismo obrero emergió durante la primera mitad del siglo XIX, ante la pasibilidad del Estado, y las leyes de accidentes de trabajo del último tercio de dicho siglo derivaban de la preocupación por la cuestión social; los seguros sociales se crearon en Alemania a finales de I88I, como resultado del Plan de Bismarch, hasta extenderse luego a toda Europa. Bismarch proponía convertir al Estado en una compañía aseguradora. Se trasladaba la técnica procedente del seguro privado a los riesgos sociales con la imposición del seguro legal y obligatorio.

En España, la historia nos ayuda a recordar que en poco tiempo hemos pasado de la beneficencia al Estado de bienestar, pasando por los seguros sociales. En el siglo XIX el Estado liberal desmanteló el sistema religioso de caridad e inició el tránsito de la caridad, como medio de asistencia social, y del mutualismo gremial o de raíz religiosa, como forma de provisión, a la beneficencia pública. Se trató de articular una política asistencial que alcanzara a erradicar la injusticia social mediante la configuración de un Estado fuerte y burocratizad que contara con un potente sistema público de financiación. Paralelamente se pedía un Estado mínimo.

Por otro lado, la implantación de los seguros sociales se realizó durante la primera mitad del siglo XX: la Ley de Accidentes de Trabajo (de I900, modificada en I922); la creación del Instituto Nacional de Previsión (I908); el Retiro obrero (I9I9), etc. La Constitución de la II República (I93I) se comprometió a instaurar una red de seguros sociales (art. 46). En los años del régimen del General Franco la normativa a ese propósito fue el Fuero del Trabajo (I938), el Seguro de Vejez (I993), el Seguro Obligatorio de Enfermedad (I942), etc.

Este desarrollo asistemático de la acción social a lo largo de los siglos se consolida a partir de la Constitución de 1978, momento en el que podemos hablar con fundamento del Sistema público de Servicios Sociales, un sistema con marcos legales diversos, y encuadrados en diferentes sistemas institucionales.

${ }^{5}$ Una lectura pormenorizada de las distintas Leyes autonómicas de Servicios Sociales nos hace ver que, al comienzo de su articulado, todas las leyes se refieren a la finalidad que tienen. Todas coinciden en atribuir como 
primera como de segunda o tercera generación, marcos normativos que utilizan el término de Servicios Sociales para referirse a ellos como un sistema público de servicios que garantiza determinadas prestaciones sociales a favor del pleno y libre desarrollo de la persona dentro de la sociedad, a promover su participación en la vida ciudadana y a conseguir la prevención, tratamiento y eliminación de las causas que conducen a su marginación y /o exclusión social. Se estructura así este tipo de servicios como un derecho legítimo de los ciudadanos a exigirlos y un deber del Estado a proporcionarlos. ${ }^{6}$

En su carácter propiamente administrativo, orgánico e instrumental el Sistema Público de Servicios Sociales es un conjunto de estructuras organizativas administrativas destinadas a dar respuesta a las necesidades personales y sociales más próximas e inmediatas a los ciudadanos o a determinados grupos (menores, mayores, jóvenes, discapacitados, etc.) con el fin de favorecer la interrelación de la persona con el medio social, de elevar la calidad de vida, promoviendo el bienestar individual y colectivo.

Los Servicios Sociales se consideran así una parte integrante de la Administración pública que, a través de unidades administrativas y equipos técnicos (organización), gestionan recursos y prestaciones (instrumentos). ${ }^{7}$

finalidad principal de los Servicios Sociales la consecución del bienestar social de la población, a la que se añaden otras finalidades, como la promoción de la autonomía personal, familiar y de los grupos humanos, la prevención de situaciones de exclusión social, la solidaridad, la cohesión social, etc.

Algunos marcos legislativos, como es el caso del País Vasco, manifiestan de forma explícita que el bienestar social no constituye una finalidad exclusiva del Sistema público de Servicios Sociales sino que este bienestar social es compartido por otros sistemas públicos de protección social de forma cooperativa y coordinada (véase el artículo 6.I de la Ley I2/2008, de 5 de diciembre, de Servicios Sociales del País Vasco).

${ }^{6}$ Las razones que avalan ese carácter público se pueden resumir en: I. Su regulación está presidida por el principio de comunidad. Son normas que imponen los poderes públicos, en uso de su plena soberanía, para conseguir la justicia y el equilibrio sociales exigidos por el Estado social de Derecho. 2. Las leyes autonómicas de Servicios Sociales hacen mención al principio de responsabilidad pública, que consiste en la adscripción de recursos financieros, humanos y técnicos, en la implantación de prestaciones públicas, y en la garantía de reconocimiento de derechos subjetivos a los ciudadanos. 3. La gestión de los servicios se encomienda a órganos, organismos públicos o entidades de Derecho público. 4. El régimen jurídico de los órganos gestores, así como el procedimiento administrativo común, tienen carácter público. 5. Las entidades privadas prestadoras de Servicios Sociales requieren autorización pública para su funcionamiento y han de estar inscritas en un registro público, condición indispensable para que puedan iniciar sus actividades. 6. Se encuentra establecido un régimen sancionador en materia de Servicios Sociales. 7. Las prestaciones de Servicios Sociales se financian mayoritariamente con cargo a recursos públicos. 8. Las garantías de los beneficiarios sobre reconocimiento de prestaciones y servicios son exigible ante el orden jurisdiccional contencioso-administrativo, cuando se trata de reclamaciones sobre actos del Estado, comunidad autónoma o entidades locales, o bien en el orden jurisdiccional de lo social si se trata de prestaciones económicas o Servicios Sociales propios de la Seguridad Social.

${ }^{7}$ DISERTACIÓN. En un esfuerzo de síntesis, M. Aguilar ha concretado que a raíz de la Constitución de I978, la relación que los ciudadanos mantienen con la Administración se materializa en:

a.- Prestaciones que constituyen un derecho subjetivo, es decir, aquellas situaciones en las que el ciudadano que reúne los requisitos de acceso puede exigir la prestación y la Administración está obligada a otorgársela. Dicho derecho está protegido por los tribunales. A su vez, los derechos pueden ser de tres tipos: i.- Derechos universales, reconocidos legalmente a todos los ciudadanos que cumplen con unos requisitos básicos de necesidad, con independencia de sus circunstancias económicas o de la existencia de aportaciones previas; por ejemplo, el derecho a la escolarización de todas las personas entre seis y i6 años de edad. 2.- Derechos contributivos, reconocidos a quienes hayan realizado una determinada aportación previa. Con frecuencia aquello a lo que se tiene derecho está modulado en función del volumen de dicha aportación; por ejemplo, el derecho a una pensión contributiva de jubilación de quien se jubile y haya cotizado un cierto número de años. 3.- Derechos de asistencia social o asistenciales, independientes de aportaciones previas pero reconocidos a quienes tienen unas circunstancias económicas que hacen que se les considere como carentes de medios suficientes. Normalmente dicho nivel de medios corresponde a aquel a partir del cual se considera que la persona podría hacer frente por sí misma a la necesidad; por ejemplo, el derecho a una pensión no contributiva de jubilación para los mayores cuyos ingresos sean inferiores a un baremo.

b.- Prestaciones que no constituyen un derecho subjetivo exigible por el ciudadano pero que responde a una obligación de los poderes públicos de atender determinadas necesidades. El ciudadano tiene la legitimidad y el 
Por otro lado, el sistema español de Servicios Sociales, o Sistema público de Servicios Sociales, comparte las peculiaridades que caracterizan a los países de la Europa meridional, un sistema que poco a poco está cambiando, modernizándose y adaptándose a las nuevas necesidades y riesgos sociales. ${ }^{8}$

Ahora bien, pese a lo dicho, tengo que resaltar que la Constitución Española de I978 no contiene expresamente el reconocimiento de un Sistema público de Servicios Sociales; más esto no significa ninguna limitación o restricción dado que ese reconocimiento se puede deducir del sentido global de la Constitución y de la interpretación de varios de sus artículos. Por ejemplo, ya en el art. I se enumera con claridad los valores en los que se asienta el Estado español: "España se constituye en un superiores del ordenamiento jurídico la libertad, la justicia, la igualdad, y el pluralismo político". El art. 9.2 indica expresamente que corresponde a los poderes públicos promover las condiciones para que la libertad y la igualdad del individuo y de los grupos sean reales y efectivas y, en consecuencia, teniendo en cuenta que los Servicios Sociales son un sector del bienestar social o un instrumento de la Política social, son un medio para hacer efectivo el derecho a vivir en una sociedad más justa o más igualitaria. El art. I4 es el que fundamenta la intervención política y administrativa a favor de grupos en situación objetiva de desigualdad por cualquier causa. Otros artículos hacen mención a los ámbitos y sectores específicos de los Servicios Sociales: el art. 39.I recoge la protección de la familia, el art. 39.2 aborda la protección a la infancia, el art. 42 se refiere a la protección de los emigrantes y retornados, el art. 49 se implica en la política pública a favor de las personas con discapacidad, el art. 50 alude a la protección a la tercera edad. El art. I48.I.2o de la Constitución señala entre otras competencias que podrán asumir las Comunidades Autónomas la "Asistencia Social".

Esta supuesta dispersión ha llevado a que algunos estudiosos y profesionales insistieran en la necesidad de que el gobierno del Estado elaborara una Ley marco de Servicios Sociales que fuera el referente para las posteriores leyes autonómicas. Pero no fue así y la historia nos recuerda que se promulgaron, y se promulgan, leyes en las distintas Comunidades autónomas y, por añadidura, en La Rioja ${ }^{9}$.

Así las cosas, el Sistema Público de Servicios Sociales en todas las Comunidades Autónomas se rige por unos principios rectores: I. Responsabilidad pública. 2.

derecho de hacer saber sus circunstancias, y la Administración la obligación de atenderlas si considera que entra en el campo de sus obligaciones de proteger un interés público, pero el ciudadano no tiene el derecho propio a exigir la prestación específica. Las prestaciones de la beneficencia pública funcionaban según este régimen, como también muchas de las rentas mínimas de inserción en España, que pueden ser o no concedidas en función de limitaciones presupuestarias o de valoración sobre su conveniencia en cada caso concreto.

c.- Prestaciones y acciones graciables o discrecionales, que la Administración puede, discrecionalmente, otorgar o no; por ejemplo, la concesión de muchas subvenciones a entidades no lucrativas es discrecional.

Fuente: M. Aguilar Hendrickson "Servicios sociales: las tribulaciones de un sector emergente", en Luis Moreno (Ed.). Reformas de las políticas del bienestar en España. Madrid Siglo XXI (2009), pág. I7I a I75.

${ }^{8}$ La aparición de nuevas necesidades sociales, nuevos factores de exclusión social, etc. hacen prever una demanda creciente de estos servicios, tanto en volumen como en diversidad, pero existe cierta incertidumbre sobre cómo se afrontará todo esto por parte de los poderes públicos pues no podemos olvidar que queda mucho camino para alcanzar el propósito universalista y para llegar a los niveles medios de gasto y cobertura de países de nuestro entorno cultural.

Estudios recientes permiten hablar de leyes de primera, segunda generación e incluso de tercera generación. En las Leyes de primera generación se observaba un predomino de la concepción pública de los Servicios Sociales, en donde el sector privado sin ánimo de lucro ocupaba generalmente un papel totalmente subsidiario y no existían casi referencias al sector de iniciativa privada lucrativa.

En las Leyes de segunda generación aparece un mayor protagonismo del sector privado, tanto del de las organizaciones no lucrativas como del sector empresarial (este aspecto es muy evidente en Galicia, Madrid y La Rioja con la opción de la relación integrada público - privado en el sistema de Servicios Sociales. De tercera serían las Leyes recientemente promulgadas. 
Universalidad. 3. Igualdad. 4. Prevención. 5. Atención personalizada. 6. Proximidad. 7. Participación ciudadana. 8. Calidad. ${ }^{\text {Io }}$

Por otro lado, las Leyes autonómicas de Servicios Sociales no son del todo uniformes al establecer qué personas, en qué condiciones y bajo que premisas, tienen derecho a tales Servicios públicos. En algunos casos se tienen en cuenta dos criterios principales: nacionalidad y residencia, en otros, se exige el criterio de residencia legal en la comunidad respectiva. En el caso de los extranjeros, lo más común es encontrar que las Leyes remitan a la legislación estatal sobre extranjería y a lo dispuesto en los Tratados y Convenios internacionales suscritos por el Estado español.

Igualmente los marcos normativos describen los derechos y deberes de las personas a los Servicios Sociales. En el capítulo de derechos, las primeras Leyes de servicios se orientan a establecer las prestaciones que podían constituir su objeto. Más adelante se entendió que los pilares de las Leyes debían de ser los derechos que tienen las personas y, en la actualidad, se configuran determinadas prestaciones de Servicios Sociales como derecho subjetivo del ciudadano, dicho de otro modo, como prestaciones exigibles por los ciudadanos a los poderes públicos. ${ }^{\text {II }}$ Bajo este prisma, son muchos los derechos que se reconocen en las Leyes autonómicas, sin perjuicio de la referencia a los derechos constitucionales. Por ejemplo, derecho a la información, a un plan de atención personalizada, acceso a los Servicios Sociales en condiciones de igualdad, a la calidad de las prestaciones o, por citar un último ejemplo, derecho subjetivo a las prestaciones por dependencia previstas en la LAPAD - Ley 39/2006, de I4 de diciembre, de Promoción de la Autonomía Personal y Atención a las personas en situación de dependencia -. En el capítulo de deberes, las leyes autonómicas contemplan, entre otros requisitos, que los ciudadanos deben cumplir las normas y requisitos establecidos en relación con las prestaciones y servicios, facilitar información necesaria y veraz sobre sus circunstancias personales, familiares o económicas, destinar las prestaciones económicas a la finalidad por la que se le concedieron o contribuir, si fuera necesario, a la financiación del coste del servicio recibido.

Otro rasgo distintivo del Sistema público de Servicios Sociales es su estructura funcional $\mathrm{u}$ organización funcional. Todas las Leyes autonómicas de Servicios Sociales hacen referencia a dos niveles de atención en que se presentan los Servicios Sociales. En el primer nivel se encuentran los llamados Servicios Sociales generales, también llamados comunitarios, de base, o simplemente de primer nivel dependiendo de la Comunidad Autónoma de referencia. En el segundo nivel se encuentran los llamados Servicios Sociales especializados, de atención especializada, de atención secundaria. Por lo demás, bajo esa heterogeneidad terminológica se esconde una misma realidad: el primer nivel, de competencia municipal, se utiliza para designar los servicios que tienen un carácter polivalente y están dirigidos a toda la población (puerta de entrada a las prestaciones del sistema para toda la población); el segundo nivel, de competencia autonómica preferentemente, da respuesta a situaciones de necesidad que requieren una especialización técnica, se ofrecen en centros y servicios específicos. ${ }^{\text {I2 }}$

\footnotetext{
${ }^{10}$ Para profundizar en estos conceptos, recomiendo la lectura de C. Alemán Bracho (coor.). Fundamentos de los Servicios Sociales. Valencia, Tirant lo Blanch, 20 оo.

${ }^{\text {II }}$ Por ejemplo, la Ley 7/2009, de 22 de diciembre, de Servicios Sociales de La Rioja, publica en el Boletín Oficial de La Rioja el 28 de diciembre de 2009, especifica que los servicios y prestaciones del Sistema Público Riojano de Servicios Sociales se configura como un derecho subjetivo, dentro del marco del Catálogo de servicios y prestaciones del mencionado sistema.

${ }^{12}$ Algunos Servicios Sociales incluidos en el segundo nivel: Familia (su finalidad es orientar y asesorar a las familias, favoreciendo el desarrollo de la convivencia, interviniendo en situaciones de conflicto y previniendo la marginación y /o exclusión social). Infancia y adolescencia (tiene por objeto el desarrollo de actuaciones para la atención social de niños y adolescentes). Personas mayores (tiene por objeto desarrollar actuaciones y establecer las condiciones de vida adecuadas de la persona mayor, fomentando su autonomía y libertad personal,
} 
Como ha destacado M. Aguilar, "si se analiza la descripción normativa de cada uno de los niveles de servicios, parecería que nos encontramos más ante dos modelos opuestos de organización que ante dos componentes de un mismo modelo" ${ }^{\text {I3 }}$. Y continua, "El nivel primario se ha desarrollado más rápidamente y parecía estar llamado a ejercer el papel central en el nuevo modelo ... Su definición genérica y su orientación generalista, sin la fijación de responsabilidades concretas, han contribuido a establecer una red de servicios bastante dispersos, carentes en general de capacidad de articular estrategias frente a problemas concretos en sus territorios. La fragmentación de una parte de la red en servicios muy pequeños (aunque varíe mucho de unos territorios y Comunidades a otros), la escasa especialización de su acción y la definición inespecífica han colocado la acción del nivel primario muy a remolque de la demanda y han favorecido su burocratización" ${ }^{14}$.

Este modelo se materializa en una organización territorial concreta. En este sentido, las Leyes autonómicas entienden por organización territorial la adscripción de centros, servicios y recursos de Servicios Sociales a un ámbito territorial determinado, de forma que sirvan, preferentemente, para la satisfacción de las necesidades de las personas residentes en ese territorio. La tendencia más reciente en las Leyes es la de establecer dos unidades territoriales de referencia: la zona básica de Servicios Sociales y el área de Servicios Sociales, que vienen a coincidir con los niveles de atención social primaria y especializada ${ }^{\text {I5 }}$.

Otros rasgos distintivos de nuestro Sistema público de Servicios Sociales son las prestaciones (prestaciones de servicios, económicas o tecnológicas), las directrices para planificar adecuadamente el sistema, la financiación ${ }^{16} \mathrm{o}$ la participación ciudadana ${ }^{\mathrm{T} 7}$.

favoreciendo la permanencia en su medio habitual de vida, etc.). Personas drogodependientes (colaboran con los servicios de salud con objeto de desarrollar programas, proyectos, etc. encaminados a la prevención, tratamiento e integración social de las personas drogodependientes, así como la dotación de personal y equipamientos adecuados a sus necesidades).

${ }^{13}$ Manuel Aguilar Hendrickson "Servicios sociales: las tribulaciones de un sector emergente", en Luis Moreno (Ed.). Reformas de las políticas del bienestar en España. Madrid Siglo XXI (2009), pág. I94

Desde el punto de vista de M. Aguilar, los problemas que se derivan de esta estructura son de distinto tipo. Por ejemplo, las dificultades para el desarrollo de políticas coherentes que supone la separación políticoadministrativa de servicios que actúan en el mismo ámbito territorial; igualmente supone una fractura de financiación Comunidades Autónomas y Ayuntamientos (al menos los grandes) se responsabilizan por separado de cada parte del sistema. De ahí se han derivado muchos conflictos, por ejemplo, en un momento como el actual de escasez de financiación en ambos niveles.

${ }^{14}$ Manuel Aguilar Hendrickson "Servicios sociales: las tribulaciones de un sector emergente", en Luis Moreno (Ed.). Reformas de las políticas del bienestar en España. Madrid Siglo XXI (2009), pág. I99

${ }^{15}$ La zona básica de Servicios Sociales se constituye por uno o varios municipios limítrofes, mancomunidades de municipios, concejos o comarcas. El área de Servicios Sociales es una estructura territorial y organizativa constituida por una agrupación de zonas básicas de Servicios Sociales en base a criterios de eficacia y eficiencia.

${ }^{16}$ Las distintas Leyes autonómicas determinan como fuentes de financiación los créditos establecidos en los presupuestos generales de la Comunidad autónoma, los créditos fijados en los presupuestos de las diputaciones provinciales o entidades similares, los créditos en los presupuestos de los Ayuntamientos o mancomunidades, los recursos de carácter extraordinario que se destinen por las Administraciones autonómicas o locales, la asignación de créditos por la Administración general del Estado (incluidos los de Seguridad Social), los precios públicos $\mathrm{u}$ otras aportaciones de las personas usuarias de los servicios y herencias, donaciones y otras aportaciones que realicen personas físicas o jurídicas de naturaleza privada.

${ }^{17}$ La participación ciudadana es un pilar básico de nuestro Sistema público de Servicios Sociales con objeto de facilitar la participación de los individuos y las organizaciones en la creación, mantenimiento, etc. del sistema. Dicha participación se concreta en la existencia de órganos colegiados (Consejo general de Servicios Sociales, Consejos locales, Consejos sectoriales de personas mayores, discapacidad, etc.) y órganos de participación en centros. Su denominación varia en función de la Comunidad autónoma de referencia. 
De todo lo dicho se desprende que, en este momento, el Sistema público de Servicios Sociales es un sistema consolidado, que forma parte del sistema más global de protección social, junto con los otros sistema de Sanidad, Educación, etc. contribuyendo al desarrollo de nuestro Estado Social y Democrático de Derecho, o dicho con otras palabras, del Estado de bienestar cuyo porvenir lo tenemos que definir entre todos. ${ }^{\mathrm{I}}{ }^{8}$

\section{II.I. De lo general a lo concreto. Algunas consideraciones sobre el Sistema Público Riojano de Servicios Sociales}

El surgimiento de los Servicios Sociales en la Comunidad Autónoma de La Rioja hasta su estructura actual es el resultado de la actuación de distintas instituciones que venían existiendo antes de la transición y el desarrollo democrático y un desarrollo normativo considerable que ser remonta a principios de los años $80 .{ }^{\text {I9 }}$ En otras palabras, es a partir de la instauración de la democracia cuando se crean los Servicios Sociales en la Comunidad Autónoma de La Rioja.

Hagamos un poco de historia. Hasta I988 la Administración Central no firmó con las distintas Comunidades Autónomas el Plan Concertado para el desarrollo de

\footnotetext{
${ }^{18}$ Ver artículo de Carmen Morán "El cuarto pilar del bienestar está cojo. Los servicios sociales carecen de financiación básica estable y de una ley estatal que los consolide - Con la crisis, la necesidad está llamando a una puerta equivocada, la del trabajador social" en El País 20/07/2009.
}

${ }^{19}$ Desarrollo normativo de Servicios Sociales en La Rioja. Ejemplos significativos:

- $\quad$ Ley 2/ I990, de io de mayo, de Servicios Sociales

- $\quad$ Orden de 4 de febrero de I992, por la que se crea el Consejo Sectorial de Tercera Edad.

- $\quad$ Orden de 20 de junio de 1996 de la Consejería de Salud, Consumo y Bienestar social por la que se crea el Consejo Sectorial de Discapacitados.

- $\quad$ Orden de 8 de abril de I997, de la Consejería de Salud, Consumo y Bienestar Social, por la que se crea el Consejo Sectorial de la Mujer.

- $\quad$ Orden de ir de febrero de I998, de la Consejería de Salud, Consumo y Bienestar Social, por la que se crea el Consejo Sectorial de Infancia y Adolescencia.

- Decreto 40 /1992, de I de octubre por el que regulan las subvenciones, conciertos y ayudas económicas individualizadas en Servicios Sociales.

- Decreto 47 /I996, de 30 de agosto, por el que se crea la Comisión Interinstitucional de Bienestar Social.

- $\quad$ Ley 5 /1998, de I6 de abril, de Derechos y Deberes de las personas usuarias, autorizaciones administrativas, infracciones y sanciones e inspección en el ámbito de los servicios sociales de la Comunidad Autónoma de La Rioja.

- $\quad$ Ley I /2002, de I de marzo, de Servicios Sociales.

- Orden I9 /2000, de 22 de diciembre, de la Consejería de Salud y Servicios sociales por la que se crea el Consejo Sectorial de Exclusión Social. Sociales.

- Decreto $46 / 2004$, de 23 de julio, por el que se regula el Consejo Riojano de Servicios

- $\quad$ Ley $3 / 2007$, de I de marzo, de Calidad de los Servicios Sociales.

- $\quad$ Orden $4 / 2008$, de 2I de abril, de la Consejería de Servicios Sociales por la que se crea la Comisión de Calidad de Servicios Sociales y se determinan su organización y funciones.

- $\quad$ Ley 7 /2009, de 22 de diciembre, de Servicios Sociales de La Rioja (Ley que deroga la Ley I/2002 de Servicios Sociales y la Ley 5/1998 de Derechos y Deberes de las personas usuarias, autorizaciones administrativas, infracciones, sanciones e inspecciones en el ámbito de los Servicios Sociales) 
prestaciones básicas de Servicios Sociales en las Corporaciones Locales, marco por el que se establecen los contenidos mínimos que garantizarán los Servicios Sociales Municipales y se buscan medios para impulsar su descentralización hasta el ámbito municipal. ${ }^{20}$

La Ley 2 / I990, de Io de Mayo, regula, mediante el sistema global de acción social, el conjunto de recursos destinados a favorecer el pleno y libre desarrollo de las personas y colectivos dentro de la sociedad y conseguir la prevención o eliminación de las causas que conducen a la marginación y a la desigualdad

Por su parte, la Ley I /2002, de I de marzo, una renovación legislativa desarrollada para promover y garantizar el derecho que tienen todos los ciudadanos a acceder a los Servicios Sociales. Se ordenará en un sistema integrado de responsabilidad pública.

La recientemente aprobada Ley $7 / 2009$, de 22 de diciembre, de Servicios Sociales de La Rioja, publicada en el Boletín Oficial de La Rioja el 28 de diciembre de 2009. Sus rasgos más destacados se concretan en los siguientes aspectos: actualizar la anterior normativa adaptándose a la nueva situación sociopolítica, económica y cultura de la Comunidad, favoreciendo la atención personalizada y ajustada a las necesidades de los individuos, familias, grupos y comunidades, una gestión que apuesta por la calidad del servicio donde las nuevas tecnologías adquieren un papel determinante ${ }^{2 \pi}$; igualmente se reconoce el derecho subjetivo a los Servicios Sociales a todos los ciudadanos con carácter universal ${ }^{22}$, ordena territorialmente el sistema con objeto de introducir cambios significativos en el ámbito comunitario, actualiza las definiciones de recursos, servicios y prestaciones y se crea el catálogo y la cartera de servicios y prestaciones del sistema. ${ }^{23}$

En suma, el Sistema Público Riojano de Servicios Sociales, enmarcado en la política general de bienestar social, coordina y colaborará con aquellos sistemas y recursos tanto públicos como privados que tengan por objetivo alcanzar mayores cuotas de calidad de vida, de ahí que también se incluya el Sistema Riojano para la Autonomía Personal y la Dependencia como parte del Sistema Público Riojano de Servicios Sociales.

Un aspecto novedoso de la actual Ley 7/2009, de 22 de diciembre de Servicios Sociales de La Rioja, es el artículo 37.I donde se determina la zona básica de actuación de los Servicios Sociales, contemplándose una ampliación razonable con respecto a la legislación anterior de la dotación mínima de un trabajador social de atención directa y comunitaria por cada cuatro mil habitantes (anteriormente el ratio se situaba en un trabajador social por cada cinco mil habitantes). ${ }^{24}$

${ }^{20}$ A falta de una Ley de Servicios Sociales nacionales el Plan concertado se convirtió en la única base de la que se disponía en las Comunidades Autónomas para configurar los Servicios Sociales.

${ }^{21}$ Recientemente el Gobierno de La Rioja puso en marcha un proyecto pionero en el ámbito de los Servicios Sociales - Protecniarioja -, un sistema telemático de gestión de los servicios sociales que mejorará la oferta de dichos servicios, la atención al usuario y el trabajo diario de los profesionales que trabajan en este ámbito.

${ }^{22}$ M. Aguilar realiza una interesante revisión histórica de la orientación universalista en su artículo "Servicios sociales: las tribulaciones de un sector emergente", en Luis Moreno (Ed.). Reformas de las políticas del bienestar en España. Madrid Siglo XXI (2009), pág. I85.

${ }^{23}$ El Sistema Público de Servicios Sociales diseñado en la Ley 7/2009, de 22 de diciembre, de Servicios Sociales de La Rioja, se regirá por los siguientes principios:

a) Universalidad. b) Responsabilidad pública. c) Protección del interés general. d) Igualdad y equidad. e) Prevención. f) Integración y normalización. g) Atención personalizada e integral y continuidad de la atención. h) Transversalidad de las políticas en materia de servicios sociales. i) Proximidad y descentralización. j) Coordinación y cooperación. k) Promoción de la iniciativa social. 1) Participación ciudadana. m) Calidad.

${ }^{24}$ Siguiendo con el esquema del Sistema público de Servicios Sociales del Estado Español, la Red Básica de Servicios Sociales Comunitarios en La Rioja - primer nivel- tiene como objetivo garantizar el acceso de los 
A destacar también en la Ley 7/2009, de 22 de diciembre, de Servicios Sociales de La Rioja, la apuesta por la calidad, dando así un marco referencial a la Ley 3 / 2007, de I de marzo, de Calidad de los Servicios Sociales, Ley que en su artículo i3.K establece un sistema de evaluación mediante el análisis de la opinión de los ciudadanos sobre los Servicios Sociales en general, y las actuaciones desarrolladas por los poderes públicos, en particular; igualmente, en el artículo 22 se describe el Observatorio de la calidad como plataforma de análisis periódico y uniforme de la percepción ciudadana sobre los Servicios Sociales en el ámbito de la Comunidad Autónoma de La Rioja e integrará a representantes de los centros, servicios o unidades administrativas prestadoras de Servicios Sociales así como a los agentes socioeconómicos que se determinen reglamentariamente.

En esta línea se puede situar mi modesta contribución al análisis de las percepciones ciudadanas sobre el Sistema Público de Servicios Sociales en La Rioja, base y fundamento del tercer epígrafe de este artículo.

ciudadanos a las Prestaciones Básicas de Servicios Sociales: Información, valoración y orientación; apoyos a la unidad de convivencia; alojamiento alternativo; prevención e inserción social.

Por su parte, en el segundo nivel de atención, Servicios sociales especializados, la ley determina que diseñaran sus actuaciones en función de las necesidades específicas que presenten los diferentes grupos o sectores de población: familia, infancia, adolescencia, personas mayores, personas con discapacidad, mujer, minorías étnicas e inmigración y otros grupos o sectores en los que se puedan manifestar situaciones de riesgo o de exclusión social

No es objeto de este artículo exponer a fondo la situación de los Servicios Sociales del primer y segundo nivel. Ahora bien, mis hallazgos empíricos derivados de la investigación Políticas de Servicios Sociales en La Rioja. Descripción, análisis, evaluación y prospectiva desde una perspectiva comparada, una investigación desarrollada gracias al apoyo económico de la Universidad de la Rioja I+D - API o8 /o9, me permiten afirmar que tenemos tantos modelos de intervención como Ayuntamientos los han podido desarrollar, lo que ha conllevado la dificultad de un desarrollo homogéneo y equilibrado del sistema. Además, he podido comprobar el progresivo incremento del gasto social y de las intervenciones sociales ligadas al mismo y se han incrementado en las dotaciones del personal para hacerse cargo de la gestión por ejemplo, trabajadores sociales.

Para los lectores interesados, recomiendo la lectura del informe de la Defensora del Pueblo Riojano Avance sobre la aplicación de la Ley de Promoción de la Autonomía Personal y Atención a las personas en situación de Dependencia en el que detalla el Estado de la cuestión. El informe completo se puede consultar en formato PDF en http://www.defensoradelarioja.com/es/?62

También es recomendable leer la entrevista realizada a la Consejera de Servicios Sociales en el periódico GENTE www.genteenlogroño.con núm. 250, del I5 al 2i de octubre de 20I0. "El desarrollo de la Ley a través del Sistema Riojano de Autonomía Personal y Atención a la Dependencia se está desplegando con éxito”, dice la Consejera. 


\section{Actitudes y opiniones sobre el Sistema Público Riojano de Servicios Sociales ${ }^{25}$}

Las actitudes y las opiniones de la población son ingredientes indispensables del sistema democrático de gobierno. ${ }^{26}$

\section{III.I. Valoración de Sistema público de Servicios Sociales}

En general, los riojanos entrevistados, sin distinción de edad o sexo, perciben el Sistema público de Servicios Sociales como un sistema que se ocupa, o se debe de ocupar, de cubrir las necesidades básicas de la población más necesitada, ahora bien, estas mismas personas entienden que muchas situaciones carenciales se quedan sin atender por parte de las instituciones encargadas de satisfacer las demandas sociales. Veamos algunos comentarios ilustrativos.

${ }^{25}$. Guión básico de los grupos de discusión para fotografiar el estado de la cuestión:

- $\quad$ Para empezar, ¿qué entienden por Sistema Público de Servicios Sociales?. ¿Qué necesidades sociales creen que cubre o debería cubrir?

- ¿Cómo valoran la situación general de los Servicios Sociales en La Rioja?.

- ¿Conocen o han oído hablar de las Leyes de Servicios Sociales en La Rioja?.

- ¿Conocen en primera persona los Servicios sociales comunitarios (o de base)?. Narración de experiencias (personales, familiares próximos o amigos)?. Concretando, ¿qué necesidades atienden o deberían atender?, ¿qué colectivos, ámbitos y sectores, atienden o deberían atender?, ¿en qué medida creen que están siendo capaces de realizar de forma efectiva la intervención social con los recursos y medios que cuentan?.

- ¿Conocen en primera persona los Servicios sociales especializados (o para colectivos específicos: drogodependientes, personas sin hogar, ...)?. Narración de experiencias (personales, familiares próximos o amigos)?. Concretando, ¿qué necesidades atienden o deberían atender?, ¿qué colectivos, ámbitos y sectores, atienden o deberían atender?, ¿en qué medida creen que están siendo capaces de realizar de forma efectiva la intervención social con los recursos y medios que cuentan?.

- ¿Conocen el sistema de financiación de los Servicios Sociales?, o dicho de otra forma, ¿quién mantiene este Servicio Público: el Estado, el Gobierno de la Comunidad Autónoma, los Ayuntamientos, las personas particulares, las empresas, las ONGs...?. ¿Cómo creen que se debería financiar el Sistema público de Servicios Sociales en La Rioja?, ¿tiene que ser una responsabilidad individual, pública o del mercado?, ¿deben pagar los usuarios por los servicios que reciben o, por el contrario, deben ser completamente gratuitos?.

- A su juicio, ¿quién debería gestionar el Sistema público de Servicios Sociales en La Rioja: el Estado, el Gobierno de la Comunidad, los Ayuntamientos, empresas privadas, las ONGs,...?.

- $\quad$ Después de todo lo que se ha hablado, ¿creen que existen diferencias o similitudes entre el Sistema Público de Servicios Sociales en La Rioja y otras Comunidades Autónomas?

- Para finalizar, ¿cuáles serían las recomendaciones o propuestas más urgentes para mejorar, fortalecer, cambiar, etc. el Sistema público de Servicios Sociales en La Rioja?

Es importante resaltar que no todos los grupos de discusión han contestado con la misma intensidad, profundidad, etc. a estas preguntas guía utilizadas por el entrevistador, en este caso, la investigadora.

G.D. = grupo de discusión $(\mathrm{I}, 2,3,4,5,6)$. Ver composición de los grupos de discusión en la nota a pie de página número 4 .

${ }^{26}$ La opinión pública no se suele formar a través de un proceso racional y reflexivo mediante el contraste de diversas informaciones y evaluaciones que hayan recibido, sino que suele forjarse a través de la adopción de evaluaciones prefabricadas que los ciudadanos reciben junto con la información.

Es un hecho comprobado que los ciudadanos forman sus actitudes individuales a partir de los agentes de socialización, como se ha reconocido desde los pioneros de la sociología, es decir, a través de la familia, el grupo de juego infantil, la escuela, la iglesia o confesión a la que se pertenezca o por citar otro ejemplo y, más modernamente, a través de los medios de comunicación. 
«Creo que, en principio, escasean...parece que lo que más se oye es el tema de las residencias para ancianos. Las plazas están muy limitadas...creo que debería haber bastante más ayuda pues hay mucha gente que necesita ayuda y no la hay» (G.D.2).

«Lo que pasa es que como los recursos son limitados...pues seguramente, hay que priorizar actuaciones $y$ dosificar, un poco, dónde se gasta ese dinero. Porque, en principio, a mi me parece que si hubiese dinero sobrante o recursos, seguramente, se podrían hacer muchas actuaciones y cubrir muchos problemas, digamos...pero... como creo que no, que el dinero es limitado» (G.D.3).

Como puede observarse, los entrevistados tienen dificultades para percibir el Sistema público de Servicios Sociales en su conjunto y, como ocurre en uno de los grupos, afloran otros sistemas como el sanitario, para enfocar un problema social susceptible de ser abordado, desde la coordinación institucional, por los Servicios sociales.

«Yo... yo hace un poco, hace un año que falleció mi padre; $y$ entonces buen, fue ingresado en el hospital y tal, y la verdad el hospital San Pedro a mi me parece un...las instalaciones son buenas, en principio, me parece que hay un poco de descoordinación porque claro, las instalaciones son buenas pues se acabó hace poco, pero, la sanidad aquí en La Rioja tiene bastante mala fama.... y, la verdad, no me pareció para tanto; un servicio más que aceptable. Con sus más $y$ sus menos, pero bueno, es más o menos lo que yo he tenido de experiencia con la sanidad; ya de abuelos y residencias de Alzheimer es que no tengo... no he tenido mucha experiencia» (G.D.3).

En un ámbito más cercano a la vida cotidiana, los encuestados se cuestionan su actitud ante las necesidades sociales.

(Grado de compromiso tenemos cada uno de nosotros) «Yo pagar mis impuestos. Y ahí te quedas» (...)

«a mí es que los voluntarios me parecen una pasada. Hoy en día, que todos son prisas, que no hay tiempo para nada, para que encima seas voluntario. Eso me parece, lo más humano (...) además, está de moda, están... promocionando el voluntariado, porque eso es un ahorro de dinero para las Administraciones» (G.D.5).

En el capítulo de las ausencias, debo decir que ningún grupo de discusión se ha detenido a considerar el aumento de la población en sí mismo como un problema o reto al cual la administración (el Sistema público de Servicios Sociales) debe hacer frente, sin matizar las importantes diferencias que supone si este aumento es de población pasiva (envejecimiento) o bien de una población mayoritariamente activa (población inmigrada). Y tampoco saben decir cómo ha ido evolucionando el sistema en el tiempo: no han podido o no han sabido realizar un análisis retrospectivo de un sistema de protección de reciente desarrollo e implantación desigual, enfrentado a demandas crecientes y a necesidades diversas por cubrir, un sistema que sigue teniendo, a pesar de su espectacular desarrollo, una posición periférica y subordinada en el conjunto de las políticas sociales.

«No si yo... vamos descontento tampoco, es que tampoco conozco mucho. Tampoco puedo hablar pero...no está tan mal... » (G.D.1).

\section{III.2. Conocimiento del marco normativo en el que se asientan los Servicios Sociales en la Comunidad Autónoma de La Rioja}

La palabra que lo engloba todo es desconocimiento. Los componentes de los distintos grupos de discusión no tienen opinión al respecto y esto es así pese a que la Ley 7/2009, de 22 de diciembre, de Servicios Sociales de La Rioja, fue presentada en distintos foros y divulgada por los medios de comunicación a la sociedad riojana. 
«No sé ni qué son los derechos subjetivos.

-ni idea...» (G.D.4).

III.3. Experiencias personales, directas o indirectas, relacionadas con los Sociales de primer nivel y segundo nivel

Es importante destacar que, en este aspecto, casi todos o todos los entrevistados comentan sus experiencias personales, directas o indirectas, relativas a distintos Servicios Sociales, ya sean comunitarios (o de primer nivel) o especializados (de segundo nivel). Los discursos hablan por sí solos.

«- Como cuáles... por ejemplo los centros del Ayuntamiento u otros...po estuve alguna vez...»(G.D.6).

«Yo sí que tuve alguna experiencia porque mi madre al quedarse viuda sí que, pues estábamos un poco perdidos en el tema de pensiones... Y fuimos al centro ... que dices tú; $\gamma$ te dan una cita previa $Y$ hay unas listas bastante grandes de gente $Y$, al final hemos acabado buscándonos la vida» (G.D.3).

«Gracias a Dios no tenemos que usarlos» (G.D.5).

«Yo fui al centro con el tema de mi madre, y no estaba masificado como ahora, tenías que esperar pero nos atendieron bien, no resolvieron gran cosa...pero bueno... igual tampoco da más de sí lo que tienen» (G.D.2).

«Yo recuerdo que hay trabajadores sociales que suelen ir a casas, con mayores a ayudarles a hacer la casa y eso.

- Pero eso no son los trabajadores sociales, son una especie de auxiliares. El trabajador social es el que gestiona el que valora...

Sé que hay algún caso que conozco, que van a ayudarle a hacerle las camas, a limpiarle.

- Eso funciona, está bien...es un parche... digo que igual es un parche. La persona que $i b a$, iba dos veces a la semana $y$, bueno, estaba bien. Soluciona algo, pero, la verdad es que tampoco mucho. Quita un poquito de carga » (G.D.4).

«Yo en ese caso también, asistencia a mayores en domicilio; lo que pasa es que tenía que hacer un papeleo tremendo para solicitar esa ayuda, para que te la concedieran y demás. La verdad es que iban poco rato, como media hora por la mañana. Luego ya por la tarde necesitábamos ayuda» (G.D.6).

«Si que pienso que no hay guarderías (...) para educación y demás, yo veo que está muy justo, muy limitado» (G.D.2).

«Creo que debería ser más rápido y con menos trámites. Ir a una sola ventanilla y que te preparasen todo. A lo mejor nosotros no tenemos ese problema porque somos personas jóvenes $y$ podemos ir a muchos sitios (...) y aunque nos estén mareando. Las ayudas deberían llegar más rápido porque cuando lo necesitas es cuando lo pides. (...) $y$, por supuesto, mirar quién necesita realmente esas ayudas, no a gente que está engañando (...)» (G.D.5).

«Yo conozco un centro de alojamiento alternativo que hay en Rodríguez Paterna que también es para gente... que ahí estuve yo, $y$ se trabaja con gente desfavorecida. 
- yo en mi casa tengo un piso de acogida de menores, que también...y una amiga nuestra trabaja también en un centro de...

- Cruz Roja, que también se hace muy buena labor. Se trabaja con niños, con familias...» (G.D.4).

«Yo de eso no conozco nada, conozco por oídas, pero no de esta Comunidad. Conozco gente mentalmente discapacitada» (G.D.1).

«Conozco una parejita, que no sé si tendría que meter mano la asistente social, no lo sé ¿no? Pero son yonquis los dos, colgadísimos, y al poco tiempo tres niños. ¿Quién regula esto? Viviendo de ocupas y tal...quién.

-Ahí irá la asistenta social cuando tenga un aviso y se los retirarán.

Son cosas, si se tendrían que encargar de estas cosas.

Respuesta: Y esos niños dónde se quedan, son adoptados o cómo.

-Hay algunos que siguen manteniendo contacto con la familia; $y$ otros, siempre menores, que si que los dan en adopción. Pero es más fácil que los tengan en pisos de acogida que...

- Será más fácil adoptar a un crío de estos que traer un chino.

-Pero tienen padres y es difícil renunciar a su hijo. (...)

- Enfrente de mi casa también tengo un piso de S.S tutelados, es que tengo de todo, en la calle vieja hay un edificio que están chavales con discapacidades $y$ hay educadores que les enseñan a administrarse, porque tampoco pueden estar solos, $y$ los veo muy bien $y$ están muy contentos con los educadores.

- Les enseñan a ser independientes. (...)

- A mí lo que me preocupa, como van pasando los años lógicamente, pues es el caso de abuelos, abuelas...que me imagino que la mayoría no tendrán familia. ¿De qué forma se pueden encargar esas personas, no sé, la Administración? Un señor mayor de 70 y tantos no creo que tenga capacidad de decir: es que voy a solicitar ¿no? De qué forma podría haber un control sobre esas personas (...) la cantidad de personas mayores que hay que dicen voy a solicitar una ayuda, ¿a quién? ¿Dónde? ¿Cuándo?...» (G.D.3).

«Bueno yo tengo que decir, que hace unos años, en mi casa pasamos una temporada fatal $y$ sí que en Cáritas, tuvimos que ir a Cáritas, me da igual... $y$, con nosotros se portaron muy bien, no nos preguntaron nada, nos dieron galletas, arroz...ahora que si están cobrando...

- Es que si viene alguien y dice dame una caja de arroz...le doy una caja de arroz, de galletas o lo que te haga falta...

- ¿Sin pedir nada a cambio?»(G.D.4).

«La gente dice es que Cáritas, es que Cáritas lo hemos pagado los demás. Llega de donde llega (...) Cáritas es de la Iglesia. Pero los alimentos no los compra la Iglesia...bueno lo de la ropa es otro tema, casi mejor no entrar porque nos desviamos. Es otro tema, que habría que hablar en profundidad el negocio que hay ahí. Lo que decía antes, creo que España es de los países que menos invierte en fines sociales; $\gamma$, claro, estamos en la cola $y$ encima estamos ya quitando, yo lo que digo $y$ propongo para el estudio. Es que lleguemos al nivel más alto de Europa y, cuando hayamos llegado, pues bajar...pero que nos dejen llegar a los niveles de Alemania, de los países nórdicos...Y 
luego tirar para abajo. Pero es que estamos a medio camino, por decir algo, estamos al $30 \%$ y ya nos quieren quitar, entonces, no sé» (G.D.3).

«A mí me toco que me echaron del edificio porque tenían que arreglarlo y no tenía dónde ir, estaba sin trabajo... fui a la asistenta social y no se movieron nada...porque no iba mal vestida, ni estaba delgada, ni iba drogada... y he visto otras cosas que muy bien... pero por qué, yo no tenía nada, ni familia... y tuve que recurrir a otra trabajadora en un centro, que me dijeron unos gitanos que lo habian hecho, $y$ por otra chica me alojaron en el piso que he dicho y fui voluntaria dando clases a niños (...) no creo que se tengan que dirigir solo a los casos...que también hay gente que no trabaja y tiene de todo (...) No se portaron bien, no solo porque seas drogadicto, gitano o inmigrante ...» (G.D.4).

«De todas formas hay muchas quejas en esto (...) subvenciones, pisos... oigo personas que piden subvenciones $y$ si no eres: extranjero; si no eres inmigrante; maltratada...

- Los casos extremos se están centrando en los inmigrantes que parece que son los que están peor...que puede ser que sí o no.

- Les están dando ayudas $\gamma$, encima, están cuidando ancianos sin contrato; $\gamma$, encima, con ayudas, porque no tienen trabajo, claro, yo también puedo trabajar en dinero negro y que me ayuden» (G.D.4).

«Conozco una chica que daba cursos en el Ayuntamiento a gente en exclusión social, $\gamma$ a la pobre muchacha le hacían cada pifia. Era jovencita, y era gente que venía rebotada del colegio, inmigrantes que no manejan muy bien el idioma...al principio le hacía mucha ilusión hasta que le robaron el teléfono...» (G.D.2).

"Yo tengo un amigo que trabajo en algo como un centro de menores aquí en Logroño $y$ también acabó estresadísimo... Y no acabó de baja...porque se fue» (G.D.1).

Del análisis de estas experiencias vitales se puede deducir que los entrevistados perciben que determinados colectivos son los principales destinatarios de las prestaciones y/o son los usuarios habituales (población con rentas muy bajas, personas inmigradas, sectores marginales, población gitana, personas con toxicomanías o alcoholismo, etc.) pero en continua competencia debido a la carencia de recursos suficientes para la protección de todos los colectivos, a resaltar la opinión de que la población inmigrante recibe más ayudas sociales

También es destacable la percepción de que hay grupos sociales que de forma sistemática no acceden a los Servicios Sociales, aunque a menudo puedan tener necesidades como es el caso de las clases medias.

La cuestión de la carencia de acceso de las clases medias a estas de prestaciones nos remite a dos elementos claves en el debate subyacente en el área de Servicios Sociales. Por un lado, apunta al carácter no universal de las principales prestaciones sociales del Estado de bienestar español y a la debilidad de los recursos públicos destinados a este ámbito, que genera tensiones y debates muy importantes alrededor de cómo se distribuyen estos recursos. La distancia entre el discurso universalita de la política de Servicios Sociales y la práctica de su oferta pública, que es básicamente insuficiente, selectiva y focalizada, contribuya a alimentar estas tensiones.

Por otro lado, apunta a otra cuestión clave: la radical invisibilidad del carácter dual del régimen de bienestar español. El modelo de políticas de bienestar español, como sucede en el resto de la Europa mediterránea, se estructura alrededor de una estrecha vinculación al mercado laboral, y por lo tanto facilita una protección intensa (a nivel de jubilación y paro) para grupos reducidos de personas trabajadoras, que a su vez coexiste con 
amplias capas de población que disfrutan de una cobertura débil, fragmentada e incluso inexistente.

\section{III.4. Valoración de las fuentes de financiación del sistema público de Servicios Sociales}

Respecto a la financiación de la Política social de Servicios Sociales, he comprobado que los miembros de los grupos de discusión son conscientes de las dificultades presupuestarias para hacer frente a todas las necesidades y demandas que sociales. Ante este hecho ven con "buenos ojos" la fórmula del copago. Sirva como ejemplo el siguiente comentario.

«- ¿Pero tenías que pagar?

Sí, claro. Se paga dependiendo de la pensión que tuviera mi madre; en base a lo que cobraba, que era lo minimo, pues tenías que pagar. Poco o mucho depende de lo que ganes. Los servicios sociales hay que pagarlos, yo creo que...no es lo que era antes» (G.D.4).

Un entrevistado comenta un suceso relacionado con el sistema sanitario riojano que invita a reflexionar sobre la percepción de la financiación de los Servicios Sociales.

"Yo he trabajado en sanidad aquí en La Rioja desde el 82...y lo que no se podía...es que una persona por el hecho de tener un conocido que lo meta en un hospital y lo dejan allá gratis como si fuera una residencia. Nosotros tuvimos enfermos, en el Hospital de La Rioja, no sé si procede si no lo quitas (- tiene sentido, sí (moderador)); una persona que tenía dinero y allí estuvo como si fuera una residencia; oye pues llévatelo a una residencia y paga una residencia...ocupa una cama de un hospital y a mí eso es que me parecía demencial. Había muchos más casos, pero ese fue de años $y$ dices...no, porque tiene dinero le das regalitos al médico o al jefe de servicio y los demás aguantamos el curro, que vale que si no está ese está otro...pero igual otro lo necesita más que ese (...) No pagó nunca nada, una residencia de ancianos tú que tienes dinero sabes que te la vas a tener que pagar (...). Otra cosa es alguien que lo necesita, pero si tienen y le reclaman... me parece bien» (G.D.3).

social.

Otros puntualizan que hay pocos servicios y poco dinero para invertir en bienestar

«En las residencias privadas... pagas todos los meses $y$ si tienes fincas las vendes (...)

- o les dejas las fincas a ellas (se refiere a las monjitas) » (G.D.6).

«Lo privado pagamos y lo público ¿que lo paguen los demás? Yo creo que tampoco es eso porque el saco al final se rompe (...)

- Yo es que nunca daría un servicio gratis. Aunque sea muy poco (...) algo testimonial pero pagar. Si no, no se valora» (G.D.5).

"No sé qué presupuestos tendrán los Servicios Sociales se van a esas minorías; $\gamma$, por eso, luego no hay más. No sé lo que costará (...)

- Tampoco hay que echarles la culpa. Igual lo que hay que hacer es repartirlo mejor, igual darles un poco menos a ellos... y para gente que paga impuestos también » (G.D.1).

«Yo creo que en España también hay poco dinero para Servicios Sociales, pues bueno ves que cuando sale en las noticias, pues Francia, Alemania... no sé si tienen más ingresos, son más ricos, o tienen otra sensibilidad social. (...) 
- Yo lo que creo, creo que, realmente...me da la sensación de que las ayudas están mal enfocadas; que da la sensación, como que, las ayudas no están para buscar una solución real para que esa gente salga adelante, se integre; sino que están para tapar agujeros y seguir adelante un poquito más (...) y eso es una solución cutre, porque estás tapando un agujero y más adelante vas a encontrar otro. Entonces, si que hay ayudas, pero no están bien administradas» (G.D.3).

"Yo creo que hay poco dinero y se va a la gente que está en más riesgo de exclusión social, o sea, están en una situación más límite. Para que coman, lo de Cáritas que te dan alimentos gratuitos a gente que no tiene ni para comer» (G.D.5).

Vinculado a esta cuestión, es llamativo el debate alrededor de los criterios específicos por los cuales se decide el acceso a servicios y prestaciones. Correspondiendo con la lógica de distribución de las prestaciones en el Estado español, las opiniones se focalizan en diferentes aspectos: si se tienen que tener en cuenta los ingresos personales o los familiares; si se parte sólo de los ingresos o bien también se toma en consideración el patrimonio acumulado especialmente en el caso de la gente mayor.

\section{III.5. Responsabilidades públicas, privadas, ONGs, etc. en la gestión de los Servicios Sociales, ¿quién lo hace mejor?}

El primer elemento a resaltar es que las políticas de Servicios Sociales en La Rioja se caracterizan por una gran fragmentación en cuanto a los agentes y proveedores de servicios. Dada la distribución de competencias en este ámbito, es destacable el protagonismo que toman los niveles de gobierno local y supralocal. Más, debido a la evolución histórica y a la debilidad de la cobertura pública que se ofrece, la presencia del sector privado (tanto mercantil como comunitario, benéfico y /o religioso) es muy importante, especialmente en ámbitos concretos como la lucha contra la exclusión social y la atención a colectivos específicos en riesgo de exclusión social, con enfermedades crónicas, sin techo, etc.

Es por eso que no debe extrañar que en los grupos de discusión salga el debate alrededor de estos elementos. Por ello, cabe destacar la valoración crítica de las relaciones que se dan entre las administraciones públicas, el sector privado mercantil y las organizaciones voluntarias llegando incluso a hablar de la desresponsabilización de la Comunidad Autónoma que lleva a un traspaso excesivo de servicios al tercer sector y a las empresas.

En cuanto al papel de las administraciones locales la percepción en general es muy positiva. Se destaca especialmente la proximidad y el papel clave que en las poblaciones pequeñas o en los barrios está teniendo la provisión de, por ejemplo, servicios como los centros de día, las becas escolares o las ayudas a los niños. Consideran que este tipo de políticas en manos de las administraciones locales comportan un acceso más fácil, más directo, con un trato más personal y de confianza, adecuado a las necesidades específicas, en definitiva, más cercano.

Sin embargo, independientemente de quien sea finalmente el actor proveedor de los servicios aparece la sensación de que hay carencias en cuanto a información. De hecho, se destaca que no sólo se trata de que haya poca información al respecto a las prestaciones existentes sino también de que los canales y mecanismos para buscarla no son muy claros y accesibles.

Otro elemento en la percepción de las políticas de Servicios Sociales en el carácter burocrático de la gestión. En este aspecto se enfatiza sobre los diferentes factores que generan una percepción kafkiana de este sector de la administración: la cantidad de trámites y papeleo necesario para acceder a las prestaciones, la carencia de coordinación 
entre administraciones y servicios, o la lentitud en las baremaciones requeridas para conocer derechos y ayudas concretas.

A continuación se pueden leer algunos ejemplos significativos de las opiniones de los ciudadanos.

«Tiene que haber dos partes unos servicios minimos que tiene que garantizar: el Ayuntamiento, la CC.AA. y luego para otros servicios que amplien un poco más, ahí entrarían los voluntarios. Pero unos servicios... a nivel de enfermos con Alzheimer, personas asistidas... todo esto tienen que garantizarlo la comunidad, lo público.

Yo creo que eso es otro debate (...) tampoco estoy muy de acuerdo por el hecho de que se privatice se...solo se vea, o solo se note que se va a ganar dinero y que te van a dar peores servicios.

- No no no , vete al Hospital de Calahorra que es fundación y mira...

Mi padre estuvo allí, y no sé lo que cobran...pero estamos encantados.

( ...)

-Hay una tendencia a privatizarlo todo, también (...)» (G.D.3).

«Hay otra cuestión que ha salido aquí...sobre la ineficacia del sector público en la gestión de los S.S. ; también se ha puesto en cuestión la eficacia de las ONG's con un cierto talante religioso etc...ahora también está muy en boga hablar de las ONG como buenas gestoras en el ámbito de los S.S. ¿qué opináis? Ya que ha salido el tema, ONG's de cualquier talante ideológico. ¿Podrían ser mejores que el Estado, mejores que los Ayuntamientos...? ¿Cómo lo veis?

- Yo es que separaría las ONG laicas, por así decirlo, y las religiosas. Y creo que hay que separarlas; yo igual es por mis experiencias...dentro de las no laicas... yo creo que las no laicas siempre funcionan de la misma manera: bajo mi punto de vista, muy criticable. Las laicas, $y$ vamos a tomar como referencia la mayor que hay en el país: Cruz Roja; yo creo que funcionan bastante bien» (G.D.1).

"Es que, en cuanto a eficacia de la Administración en Servicios públicos frente a las ONGs, la verdad, es que desconozco lo que hacen las ONGs. Pero me imagino que de algún modo se financiaran de la propia Administración.

-Por ahí han salido bastantes corruptelas, y la gente, la verdad es que desconfía. (...) hay que creer en el buen hacer de ellos, que al fin y al cabo, están haciendo algo que debiera hacer el Estado; y ya que lo hacen, no por eso vamos a fomentar que trapicheen con dinero, por supuesto que no, ha habido casos... tengo un amigo...que hace proyecto y le pagan. Va al extranjero y lleva una vida muy perra (...) se está planteando dejarlo, y eso que le pagan, porque llegas a una edad en la que dices (...)» (G.D.3).

«Yo tengo una amiga, que pidió una ayuda de esas (ayudas por estar impedido) Y yo sé que la pidió en el Ayuntamiento. Y veo en el médico de cabecera personas que llevan un papel, $y$ creo, que el médico da un papel para que vayas al Ayuntamiento firmado por él, de que necesitas una ayuda social. Y el Ayuntamiento, no sé a través de qué, si S.S. te desvía para empezar a preparar las cosas y te suele mandar una persona a casa. Estoy un poco perdida, sé que funciona un poco así.

- Yo creo que el Ayuntamiento y la Comunidad tienen que estar unidos» (G.D.4).

«Moderador: en esa línea, los Servicios Sociales son competencia de los Ayuntamientos $\gamma$ de las CC.AA. ¿qué les podemos proponer al Gobierno Autonómico y a los Ayuntamientos de la CC.AA.? 
Respuesta: pues yo al Gobierno de La Rioja, le propondría que hiciera alguna...tanto centros para disminuidos y tal, pero que los hiciera él; $y$ que, desde luego, no se los de a gestionar a empresas. Voy a ser duro, que sí, ponen unos criterios...pero al final la empresa esa la gestiona fulanito que es amigo del que está gobernando en ese momento. Porque entonces, nos va a costar " $x$ " pero ese " $x$ " que nos cuesta...una parte de ese " $x$ " se lo va a quedar la empresa, su amigo, con lo cual no estamos invirtiendo eso, estamos invirtiendo eso menos lo que se queda el otro, porque la gestión el que gestiona no gestiona gratis. Creo que la sociedad lo tiene muy claro si tú lo privatizas, lo externalizas (...) si público te cuesta 20 y lo externalizas y te cuesta 20, está claro que se va a gastar 18 y 2 se va a quedar él, él o ella, la empresa. Me refiero a la Comunidad porque las residencias y eso son más de la Comunidad» (G.D.3).

\section{III.6. Análisis comparado del sistema y propuestas de mejora}

Lamentablemente en los discursos de los grupos de discusión apenas existen comparaciones con otras comunidades autónomas, Europa o el mundo, quizás por desconocimiento o porque a los ciudadanos les cuesta tener una visión global de las cosas, prefieren centrarse en lo cotidiano, lo próximo (tema que podría ser objeto de investigación en futuros trabajos).

«Moderador: Ese análisis comparado me parece muy adecuado, podemos seguir en esa línea. Habéis hablado de Navarra.

- Es que lo ideal sería tener conocimiento y datos. Que fuera algo... tampoco vamos a hablar de oídas porque nos podemos equivocar (...)» (G.D.3).

Por último señalar que a los entrevistados, mayoritariamente, les cuesta pronosticar el futuro o plantear propuestas de cambio y transformación social del Sistema público de Servicios Sociales. No obstante, del análisis de los discursos se pueden extraer algunas propuestas interesantes y significativas. Los discursos son ilustrativos de lo que digo.

«Bueno, yo creo que lo ideal es que hubiese empleo, pero para alcanzar una situación ideal hace falta dinero. Yo, en mi experiencia personal (...) veo que hay mucha gente mayor; porque claro, nuestra sociedad tiende a envejecer. Entonces, la gente mayor se encuentra con trámites y papeleos que no hay quien se entere. Hace falta más asistentes sociales motivados para ayudar a toda esa gente» (G.D.1).

«A mí me gustaría una gestión pública $y$, por profesionales, que no estuvieran los políticos tan metidos en esto, que la gente que lo gestione fuera profesional de eso. (...) no cambiamos de color y cambiamos todo esto (...)» (G.D.3).

«Aparte de reducir la burocracia, poner una oficina única en la que se haga todo»

«Anticiparse a lo que viene, en vez de esperar a que llegue. Educar a las personas de cierta edad, que ahora las familias de ahora no son como las de antes con 6 o 7 hijos que mantenían a los padres...económicamente no va a llegar el Estado a satisfacer completamente; no sé, educar a esas personas a que convivan con otros vecinos, compartirse y apoyar» (G.D.2). mayores.

«Una comunidad...una urbanización que van a hacer aquí de casas pero con servicios a

Algo así.

-Con una cocina común, espacios comunes...para gente válida claro. 
- Yo patrocinaría la autonomía de esos mayores. Con servicios o asistentes que no los alejen de su entorno. Y el Gobierno de La Rioja tiene que invertir bastante más" (G.D.5).

«Todos los servicios, aunque sean de la Administración y pagados por los impuestos, hay que pagar un mínimo para que las personas no abusen. Las pensiones mínimas subirlas, las máximas congelarlas... una pensión de 2500 euros...que viene del Estado, que es social, no es muy social» (G.D.3).

En definitiva, las visiones de futuro son contradictorias. Mientras que en algunos grupos de discusión se considera que esta política seguirá mejorando, ya sea porque las necesidades y demandas siguen creciendo o porque el alargamiento de la esperanza de vida hará que esta política se tenga que priorizar; en otros casos, predomina una previsión negativa, destacando que será difícil desarrollar más los Servicios Sociales en un momento de crisis económica como el actual.

\section{Conclusiones}

En este artículo, además de revisar el desarrollo del Sistema público de Servicios Sociales en España y en La Rioja, me he aproximado - a modo de fotografía de la realidad a las percepciones (opiniones y actitudes) de los ciudadanos riojanos sobre la Política de Servicios Sociales en la Comunidad Autónoma.

De forma muy resumida, los entrevistados consideran que lo que está en juego es el debate político de fondo alrededor de la cuestión de quién tiene derecho a qué y cómo se puede conseguir un Sistema público de Servicios Sociales para distintos colectivos y grupos en un momento histórico en el que está en juego la sostenibilidad del sistema. ${ }^{27}$

La necesidad de optimización de los recursos sociales, la distribución territorial equilibrada de los mismos, las garantías de calidad básica para los ciudadanos y la propia seguridad para los gestores y responsables son, entre otros, aspectos que tendrán que ser atendidos en la Comunidad Autónoma de La Rioja con objeto de prestar, o seguir prestando, servicios de calidad a sus ciudadanos.

Comparar lo que se dice que se va ha hacer con lo que realmente se ha hecho o dejado de lado, puede ser objeto de otro estudio comparativo y de evolución del Sistema público de Servicios Sociales en La Rioja, como también lo sería comparar los objetivos del programa de un partido político o de otro, con los objetivos, por ejemplo, de los discursos de investidura y comparar después con la realidad construida o modificada. Pero esto, como diría Kipling, «es otra historia». ${ }^{28}$

${ }^{27}$ Debate candente. Recomendable la lectura del artículo de Teri Sáenz y Roberto G. Lastra, «El presupuesto de La Rioja cae un 6,4\% por la crisis», Diario de La Rioja I6/ı/20ı。.

En la actualidad nos encontramos con que el envejecimiento de la sociedad, el aumento de la complejidad social y de la demanda de la ciudadanía, la llegada de población inmigrada y el debilitamiento de las redes familiares y comunitarias de apoyo son los principales elementos que ponen en tensión los regímenes de bienestar vigentes. En consecuencia, el debate sobre la definición de los sujetos de derechos se sitúa en el marco de recursos percibidos como escasos y de lucha de los colectivos por los mismos y se vincula directamente a otro elemento: la financiación de las políticas sociales y la contribución económica de las personas (vía cotización) y su acceso a los servicios.

${ }_{28}$ «Otra historia» que debemos construir en La Rioja ya sea desde las instituciones públicas, privadas, mixtas o, porque no, desde nuestra Universidad como lugar en el que se reflexiona, analiza, compara, explica, etc. la realidad social.

En este sentido, creo útil y necesario, impulsar con fuerza desde el Departamento de Derecho una línea de trabajo e investigación en este ámbito, formando becarios, dirigiendo investigaciones, etc. de valor incuestionable para mejorar nuestro sistema público de bienestar social, en general, y de Servicios Sociales, en particular. 


\section{Bibliografía.}

AGILAR HENDRICKSON, M., «Los servicios sociales: las tribulaciones de un sector emergente», en Moreno Fernández, Luis (ed.), Reformas de las políticas de bienestar en España, Madrid, Siglo XXI, 2009, págs. I7I206.

ALEMAN BRACHO, C. (Coord.), Fundamentos de los Servicios Sociales. Valencia, Tirant lo Blanch, 2oio.

ALEMAN BRACHO, C. Y FERNÁNDEZ GARCÍA, T., Política social y Estado de Bienestar. Valencia, Tirant lo Blanch, 2006.

ALEMÁN BRACHO, C. Y GARCÍA SERRANO, M., Servicios sociales sectoriales. Madrid, Edit. Universidad Ramón Areces, 2005.

ARRIBA, C., CALZADA, I., DEL PINO, E., Las actitudes de los españoles hacia el estado del bienestar (1985-2005), Madrid, Centro de Investigaciones Sociológicas (Opiniones y actitudes, 55) (2006)

BUENO ABAD, J. R., Los Servicios Sociales como sistemas de Protección Social, ed. Nau Llibres, Valencia, I992.

CASADO, D., Reforma política de los servicios sociales, Madrid, CCS, 2002.

CASADO, D. Y GUILLÉN, E., Manual de Servicios Sociales, Madrid, CCS, 200 I.

ESTIVILL, J., "Políticas sociales en la Unión Europea en los albores del nuevo milenio", Revista del Ministerio de Trabajo y Asuntos Sociales, 32, 2002.

GALLEGO, R., GOMÀ, R. SUBIRATS, J. (editores), Estado de bienestar y comunidades autónomas: la descentralización de las políticas sociales en España, Madrid, Tecnos: Universitat Pompeu Fabra, 2003

GARCÉS FERRER, J., Sistema político y administrativo de los Servicios sociales, Valencia, Tirant lo Blanch, I995.

GARDE, J. A., Políticas sociales y Estado de bienestar en España, Madrid, Fundación Hogar del Empleado y Editorial Trotta, I999.

GUTIÉRREZ RESA, A. Y GARCÉS FERRER, J., Los Servicios Sociales en las Comunidades y Ciudades Autónomas, Valencia, Tirant lo Blanch, 2000.

GUILLÉN, E. Y OTROS, “Los cambios legislativos recientes en materia de servicios sociales, en Casado, D. y Fantova, F. (eds.), Perfeccionamiento de los servicios sociales en España, Madrid, FOESSA - Caritas, 2007.

MINISTERIO DE TRABAJO Y ASUNTOS SOCIALES, Política social de la Comunidad Europea, Madrid, Ministerio de Trabajo y Asuntos Sociales, 2002.

MUÑOZ MACHADO, S. Et al., Las estructuras del bienestar, Madrid, Civitas, I997.

PÉREZ SÁNCHEZ, M. (ed), Análisis de Políticas Públicas, Granada, Editorial Universidad de Granada, 2006.

RODRIGUEZ CABRERO, G., El Estado del bienestar en España: debates, desarrollo y retos, Madrid, Fundamentos, 2004 .

VIDAL FERNÁNDEZ, F., V Informe de políticas sociales. La exclusión social y el Estado de Bienestar en España, Barcelona, Fundación Hogar del Empleado, 2006.

VV. AA., Administración Social. Servicios de Bienestar Social, Madrid, Ed. Siglo XXI, I996. 